\title{
CONSUMO ALIMENTAR DE ESTUDANTES UNIVERSITARIOS DO SEXO FEMININO
}

\author{
Elizabeth Fujimori : \\ Hisako Shima** \\ Maria Josefina Leuba Salum***
}

FUJIMORI, E.; SHIMA, H.; SALUM, M.J.L. Consumo alimentar de estudantes universitários do sexo feminino. Rev. Esc. Enf. USP, São Paulo, 20(2):115-124, 1986.

Realizou-se um estudo dietético com o objetivo de caracterizar o padräo nutricional de estudantes universitários do sexo feminino, utilizando-se o método de pesagem direta dos alimentos. Os resultados revelaram deficiência vitaminico-mineral (predominantemente de ferro, cálcio e tiamina) na dieta, quando comparados com as recomendaçōes dietéticas.

\section{INTRODUÇÃO}

O inquérito alimentar é um instrumento essencial na avaliação das condições nutricionais do indivíduo ou de grupos da comunidade. Fornece um perfil do hábito alimentar, servindo como um dos indicadores do nível sócio-econômico, mas, principalmente, indica deficiências nutricionais que poderão advir de hábito alimentar inadequado.

No Brasil, pouco se sabe sobre o hábito alimentar de estudantes universitários do sexo feminino; praticamente inexistem estudos nesse sentido, provavelmente devido ao fato dos estudantes universitários não constituirem, primordialmente, um grupo vulnerável a deficiências nutri cionais; no entanto, muitos trabalhos realizados em outros países mostram que essa população pode estar sujeita a carências nutricionais por consumir dietas indequadas, tal qual grupos de outros segmentos da população (DRISKELL et alii, 1979; OSTROM \& LABUZA, 1977; MAEKAWA et alii, 1977; ISHIGAKI \& SUZUKI, 1975; TALEBANY, 1981).

A mulher estudante universitária, normalmente se encontra em idade fértil. $O$ organismo feminino, especialmente na idade fértil, não pode

* Enfermeira. Auxiliar de Ensino do Departamento de Enfermagem Médico-Cirúrgica da Escola de Enfermagem da USP - disciplina Nutrição o Dietética Aplicadas à Enfermagem.

* Nutricionista. Doutor em Ciência dos Alimentos. Professor Assistente Doutor do Departamento de Enfermagem Médico-Cirúrgica da Escola de Enfermagem da USP - disciplina Nutrição e Dietética Aplicadas à Enfermagem.

*** Enfermeira. Doutor em Ciências. Professor Assistente Doutor do Departamento de Enfermagem Médico-Cirúrgica da Escola de Enfermagem da USP - disciplina Nutrição $\theta$ Dietética Aplicadas à Enfermagem. 
prescindir de nutrição adequada, uma vez que precisa estar apto para enfrentar o processo gestacional, modificação fisiológica comum ao seu ciclo vital. A Organização Mundial de Saúde (1970) tem enfatizado em suas recomendações que, para a boa evolução da gestação, o ideal seria a futura mãe nunca ter sofrido desnutrição; pode-se afirmar que também a obesidade é uma pré-condição indesejável para o bom andamento do processo gestacional.

Nos paises do terceiro mundo, as carências nutricionais são problemas freqüentes, pois, grande parte de sua população vive em situação de miséria que impede a alimentação adequada. Contrastando com esse fato, as populações mais privilegiadas têm, na obesidade, um problema que vem assumindo proporções cada vez maiores e constitui preocupação de muitas mulheres a partir da adolescência e mesmo durante a maturidade. Nesse caso, como ressaltam ISHIGAKI \& SUZUKI (1975), muitas jovens reduzem drasticamente o consumo alimentar com o objetivo de reduzir o seu peso corporal; isso pode fazer com que o consumo de nutrientes permaneça muito aquém das recomendações nutricionais preconizadas, podendo também levar a deficiências nutricionais.

Realizamos o presente estudo com o objetivo de melhor caracterizar o padrão alimentar de um grupo de estudantes universitárias e verificar se haveria existência de excessos ou deficiências nutricionais que necessitariam de correção adequada.

\section{METODOLOGIA}

\section{População}

Fizeram parte do estudo 47 estudantes do sexo feminino (idade média de 19,8 anos) matriculadas no $3^{\circ}$ semestre do curso de Graduação em Enfermagem e Obstetricia de São Paulo.

Estudo dietético

$O$ inquérito alimentar foi realizado segundo a técnica de pesagem direta dos alimentos e líquidos consumidos. Para isso, utilizamos balança marca AGRAM com divisões de $1 \mathrm{~g}$ e carga máxima para $1050 \mathrm{~g}$. As estudantes foram divididas em oito grupos (A, B, C, D, E, F e G) e cada um registrou o consumo alimentar de 3 ou de 4 dias consecutivos. O grupo A colheu seus dados de $5^{a}$ feira a domingo, o grupo B de $2^{a}$ a $4^{3}$ feira, o grupo $\mathrm{C}$ de $5^{a}$ a domingo, e assim sucessivamente, de forma que, no final, obtivemos o registro do consumo alimentar de um total de 25 dias consecutivos. Para a análise centesimal de cada estudante, utilizou-se a Tabela de Composição dos Alimentos da Fundação Instituto Brasileiro de Geografia e Estatística (1977); os dados não encontrados nessa tabela foram retirados de outras fontes (BURTON, 1979; KAGAKU GIDYTSU CHO SHIGEN CHOSA KAI, 1979). A adaquação do consumo de energia e nutrientes de cada estudante foi verificada confrontando-se os dados (médias de 3 ou 4 dias) de consumo com as recomendações do Food and Nutrition Board do National Research Council (1980), (porcentagem de adequação $=$ consumo $/$ recomendação $\times 100$ ). 


\section{Estudo antropométrico}

Verificou-se a estatura, o peso corporal e a circunferência do punho de cada estudante. Para a tomada do peso corporal foi utilizada balança de marca Filizola série 3134 , com capacidade para $150 \mathrm{~kg}$, e escala com divisões de $100 \mathrm{~g}$. As estudantes foram pesadas sem agasalhos e sem calçados, sendo a balança aferida antes de cada pesagem. A medida da estatura foi realizada após a pesagem, no mesmo equipamento: as estudantes foram colocadas eretas, com os pés paralelos e com as nádegas e o occipital tocando a haste. A medida da circunferência do punho foi tomada logo abaixo da apófise estilóide, no nível da prega do punho da mão direita. Esse dado foi utilizado na classificação da estrutura óssea, conforme descrevem GRANT et alii (1981) e o peso ideal pôde assim ser determinado segundo o critério desses mesmos autores.

\section{RESULTADOS E DISCUSSÃO}

Apresentamos na tabela 1 as médias de idade, estatura e peso corporal da população estudada.

TABELLA 1

MEDIAS ( $\bar{x}$ ), DESVIOS PADRAOO (s) E VALORES MINIMOS E MAXTMOS DA IDADE, ESTATURA E PESO CORPORAL DA POPULAÇAO ESTUDADA.

\begin{tabular}{lcccc}
\hline $\begin{array}{l}\text { Idade, estatura } \\
\text { O peso corporal }\end{array}$ & $\overline{\mathbf{x}} \pm \mathrm{s}$ & \multicolumn{2}{c}{ minimo } & máximo \\
\hline Idade (anos) & $19,8 \pm 1,9$ & 18 & 29 \\
Estatura (cm) & $1,58 \pm 0,1$ & 1,46 & 1,73 \\
Peso corporal $(\mathrm{kg})$ & $50,9 \pm 6,9$ & 40,0 & 72,9 \\
\hline
\end{tabular}

Apesar da grande variação observada na idade (18 a 29 anos), notamos que a média foi bem próxima do valor mínimo, já que a maioria das estudantes ( $96 \%$ da população) tinha de 18 a 32 anos.

No que se refere à estatura média da população, observamos que ela é praticamente o percentil 50 do padrão Santo André Classe IV para adolescentes do sexo feminino, de 19 anos e 8 meses (idade média da população) (MARCONDES, 1982).

$O$ peso corporal médio da população foi coincidente com o peso ideal, segundo a análise proposta por GRANT et alii (1981); no entanto, comparando com o padrão para adolescentes de 19 anos e 8 meses da tabela de Santo André Classe IV (MARCONDES, 1982), o peso médio da população estudada fica em torno do percentil 35.

A tabela 2 mostra a distribuição da população de acordo com a sua adequação de peso corporal (Peso corporal/peso ideal $\times 100$ ). Verificamos que a maior parte da população estudada apresentava peso adequando; a ocorrência de magreza (menos de 90\% de adequação) e de 
exesso de peso (mais de $110 \%$ de adequação) foi semelhante à que OSTRON \& LABUZA (1977) encontraram em uma população de estudantes universitários norte-americanos. No entanto, vale a pena ressaltar que a estudante mais magra apresentou peso corporal $20 \%$ abaixo da tabela de GRANT et alii (1981) e a mais gorda, 25\% acima.

TABEILA 2

DISTRIBUIÇÃO DA POPULAÇAO ESTUDADA SEGUNDO A ADEQUAÇAO DO PESO CORPORAL.

\begin{tabular}{lll}
\hline $\begin{array}{c}\text { Adequacá do } \\
\text { peso corporal }\end{array}$ & \multicolumn{2}{c}{ Populaça } \\
\hline menos de $90 \%$ & 21 & 10 \\
90 a $110 \%$ & 62 & 29 \\
mais de $110 \%$ & 17 & 8 \\
\hline
\end{tabular}

O consumo médio de energia e de nutrientes é apresentado na tabela 3 e na figura 1, onde pode ser observada a porcentagem média de adequação da energia e dos vários nutrientes consumidos pela população.

TABELA 3

MADIA ( $\bar{x}$ ), DESVIO PADRAO ( $s$ ), VALORES MINIMO E MAXTMO DA PORCENTAGEM DE ADEQUAÇAO DE ENERGIA E NUTRIEINTES CONSUMIDOS PELA POPULAÇAO ESTUUDADA.

\begin{tabular}{lcccc}
\hline Componentes & Recomendaçoes* & $\begin{array}{c}\text { Consumo } \\
(\overline{\mathbf{x}} \pm \mathrm{s})\end{array}$ & $\begin{array}{c}\text { Porcentagem } \\
\text { mínima }\end{array}$ & $\begin{array}{c}\text { dé adequaçá } \\
\text { máxima }\end{array}$ \\
\hline Energia (Kcal) & 1935 & $1990 \pm 439$ & 56 & 187 \\
Proteina (g) & 40,7 & $74,8 \pm 19,9$ & 106 & 388 \\
Vitamina A (mmg) & 800 & $759 \pm 1041$ & 15 & 919 \\
Vitamina C (mg) & 60 & $99 \pm 7$ & 17 & 600 \\
Tiamina (mg) & 1,10 & $0,91 \pm 0,28$ & 17 & 489 \\
Riboflavina (mg) & 1,30 & $1,28 \pm 0,58$ & 48 & 295 \\
Niacina (mg) & 14,0 & $17,0 \pm 6,8$ & 61 & 314 \\
Cálcio (mg) & 800 & $607 \pm 276$ & 30 & 189 \\
Ferro (mg) & 18,0 & $13,1 \pm 4,0$ & 38 & 142 \\
\hline
\end{tabular}

* Extraido de FOOD AND NUTRITION BOARD (1980).

Analisando a adequação média do consumo de nutrientes (figura 1), observamos que o cálcio, o ferro e a tiamina encontram-se com porcentagem de adequação inferior a $90 \%$. De outro lado, notamos aumento considerável da porcentagem de adequação para proteínas e vitamina C. 


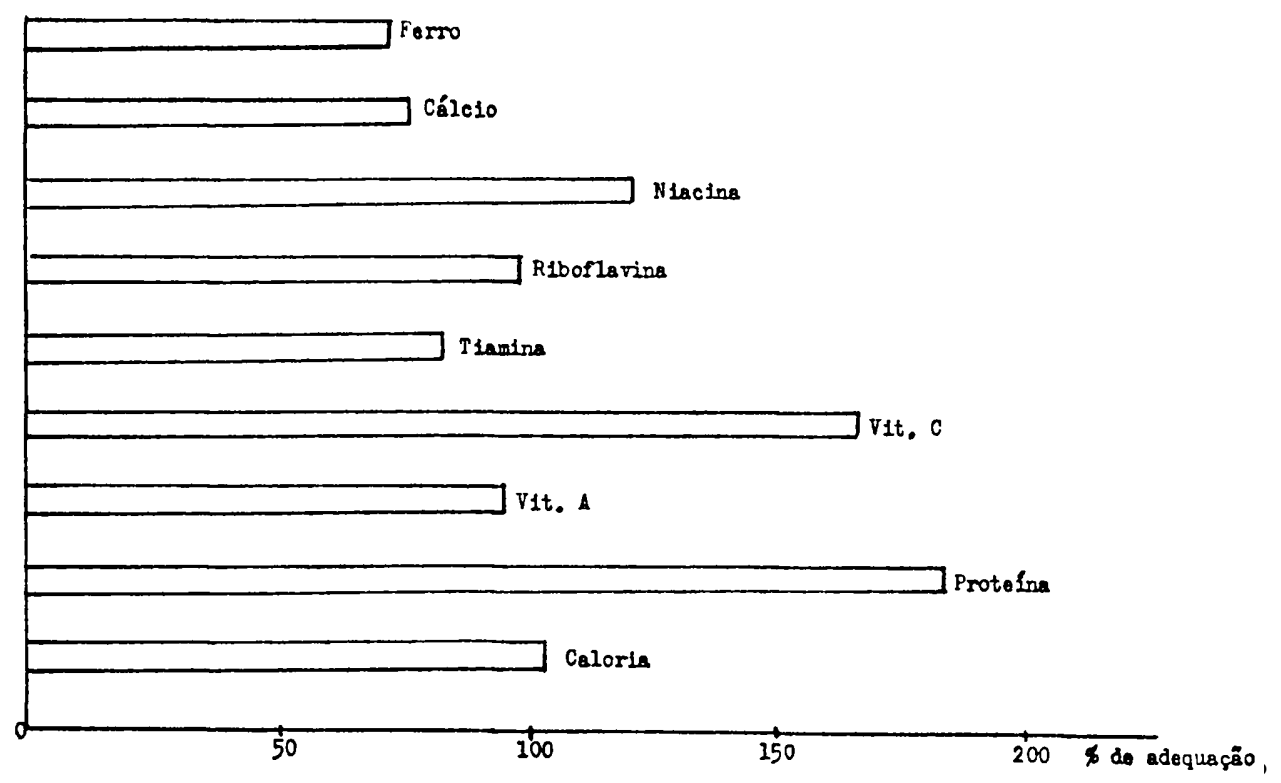

Figura 1 - Adequagăo média do consumo, em porcentagem, da população estudada.

Esses dados coincidem em parte com os obtidos por ISHIGAKI \& SUZUKI (1975) e MAEKAWA et alii (1977) que, ao analisarem a ingestão de nutrientes e o consumo calórico de estudantes universitárias japonesas, encontraram baixo consumo de cálcio e ferro e elevado consumo de proteínas e vitamina $C$; diferentemente do que observamos, verificaram, além disso, baixo consumo médio de vitamina $\mathrm{A}$.

Já DRISKELL et alii (1979), avaliando o estado nutricional de estudantes universitários norte-americanos, obtiveram resultados referentes ao consumo de ferro, tiamina, proteína e vitamina $\mathrm{C}$, com os quais os nossos dados coincidem.

Quanto ao consumo calórico, verificamos no presente estudo que $26 \%$ da população consumiu menos de $90 \%$ de sua provável necessidade calórica e $38 \%$ consumiu calorias a mais, isto é, mais que $110 \%$ da sua provável necessidade calórica.

Merece consideração, ainda, a distribuição percentual das calorias segundo os nutrientes energéticos: em média, $50,5 \%$ das calorias totais provinham dos glicídios, $35,7 \%$ dos lipídios e $15 \%$ das proteínas. A distribuição dos nutrientes energéticos na dieta observada no presente estudo foi similar àquela encontrada por OSTRON \& LABUZA (1977).

O Committee on Dietary Allowances do FOOD AND NUTRITION BOARD (1974) afirmam que a proporção de calorias derivada dos lipídios não deve exceder $35 \%$. Assim sendo, o consumo médio de $78,9 \mathrm{~g}$ de lípides pela população estudada, que corresponde a $35,7 \%$ das calorias totais consumidas, poderia ser considerado praticamente adequado. 
Os dados da tabela 3 e da figura 1 indicam que o consumo protéico excedeu em muito a recomendação de $0,8 \mathrm{~g} / \mathrm{kg}$ de peso corporal por dia. Com relação à porcentagem do total calórico que é fornecido pelas calorias protéicas $(15 \%)$, verificamos que ela coincide com o valor máximo encontrado por TAGLE (1981), em diferentes dietas humanas.

A Análise da procedência alimentar da proteína consumida (se de origem animal ou vegetal) revela que quase $2 / 3(63 \%)$ foi de origem animal, atendendo, portanto, à recomendação de que pelo menos $1 / 3 \mathrm{da}$ proteína dietética seja de alto valor biológico (INSTITUTO DE NUTRICIÓN DE CENTRO AMERICA Y PANAMA, 1966).

Elevado consumo de proteínas de origem animal também foi observado por MAEKAWA et alii (1977); esses autores verificaram que 55\% do total de proteínas consumidas pelas estudantes japonesas foi de origem animal.

Sabe-se que consumo de alimentos de origem animal mantém estreita correlação com o nível sócio-econômico da população. Em 1976, NAKAMAE verificou que os estudantes da Escola de Enfermagem da Universidade de São Paulo provinham de extratos sociais relativamente elevados; aparentemente, a procedência social dos estudantes não se alterou de lá para cá, pois constatamos consumo médio de carnes e ovos de $174,0 \mathrm{~g}$ por dia e, de leite e derivados, de $197,5 \mathrm{~g}$ por dia.

Os dados da figura 1 sugerem não haver deficiência pronunciada no consumo dos vários nutrientes; no entanto, ao analisarmos a porcentagem de estudantes que apresentam consumo inferior a $90 \%$ de adequação, o problema assume nova feição (tabela 4).

No que toca à vitamina $\mathrm{A}$, poderíamos incorrer num erro analítico se considerássemos que o consumo médio praticamente adequado (tabela 3 e figura 1) estivesse representando o comportamento alimentar adotado pela maioria da população estudada. $\mathrm{Na}$ realidade, observamos, pela tabela 4, que a média foi alcançada como resultado do consumo bastante exagerado desse nutriente por uma minoria da população (houve quem consumisse $919 \%$ da recomendação). A grande maioria (70\%) apresentou consumo inferior à recomendação; observamos, inclusive, níveis inadmissíveis de consumo - $15 \%$ da recomendação; esses achados sugerem que a deficiência de vitamina $A$ pode constituir um dos problemas nutricionais nessa população.

As fontes mais ricas de vitamina $A$ na dieta das estudantes que consumiram além da recomendação foram fontes convencionais como ovo, cenoura, leite, espinafre, couve, caqui e batata doce; no entanto, o alimento responsável pelos $919 \%$ de adequação em uma estudante foi o fígado.

Os resultados que OSTRON \& LABUZA (1977) encontraram para a vitamina $\mathrm{A}$, em estudantes universitários norte-americanos, não diferem do observado neste estudo: encontraram, como porcentagem média de adequação, o indice de $94 \%$, mas a maioria da população consumiu 
TABELA 4

DISTRIBUIÇAO PERCENTUAL DA POPULAÇÃO ESTUDADA, SEGUNDO A PORCENTAGEM DE ADEQUAÇÃO PARA OS NUTRIENTES.

\begin{tabular}{lcccc}
\hline Nutrientes & $\begin{array}{c}\text { menos de } \\
\text { 90 adequacão }\end{array}$ & $\begin{array}{c}\text { 90-110\% } \\
\text { 90\% de adequacão }\end{array}$ & $\begin{array}{c}\text { 110\% } \\
\text { mais de adequação }\end{array}$ \\
\hline Cálcio & 66 & 19 & 15 \\
Ferro & 81 & 13 & 6 \\
Vitamina A & 70 & 11 & 19 \\
Vitamina C & 34 & 4 & 62 \\
Tiamina & 62 & 23 & 15 \\
Riboflavina & 51 & 24 & 25 \\
Niacina & 23 & 26 & 51 \\
\hline
\end{tabular}

menos de $80 \%$ da recomendação, sendo que $1 / 3$ deles ingeriram menos de $60 \%$; os estudos de MAEKAWA et alii (1977) e ISHIGAKI \& SUZUKI (1975) relatam, por sua vez, baixo consumo médio de vitamina A, generalizado, entre estudantes universitárias japonesas.

As vitaminas $\mathrm{C}$ e do complexo $\mathrm{B}$ são outros nutrientes cujos dados médios de consumo precisam ser analisados com certo cuidado.

Embora o consumo médio de vitamina $\mathrm{C}$ tenha atingido $166 \%$ da recomendação (figura 1), esse dado resultou do fato de que $62 \%$ da população estudada apresentou consumo maior do que o recomendado, como pode ser observado na tabela 4; notamos, também, que apenas $4 \%$ da população consumiu quantidades adequadas, e que $34 \%$ da população apresentou consumo inferior à recomendação.

Outros autores, trabalhando com estudantes universitárias, registraram, sistematicamente, consumo elevado de vitamina $C$, sendo que alguns deles relatam haver, também, grande variação no nível de consumo (OSTRON \& LABUZA, 1977; DRISKELL et alii, 1979; MAEKAWA et alii, 1977; ISHIGAKI \& SUZUKI, 1975).

Quanto às vitaminas do complexo $\mathrm{B}$, se por um lado o consumo médio de riboflavina e niacina se apresentaram aparentemente adequados, de outro lado, o consumo médio de tiamina atingiu apenas $83 \%$ da recomendação (figura 1). Por trás desses dados, o que se verifica, de fato (tabela 4), é que grande parte da população consumiu menos que $90 \%$ da recomendação de tiamina e riboflavina, o que não ocorreu com a niacina. Devemos ressaltar que esses resultados são bastante similares aos relatados por DRISKELL et alii (1979).

Com relação à ingestão de cálcio, notamos, na figura 1 , que o consumo médio foi de apenas $3 / 4$ da recomendação. O problema é bastante sério, como pode ser observado na tabela 4, já que esse baixo consumo 
resultou do fato de que $66 \%$ da população sequer atingiu a recomendação mínima diária do mineral.

OSTRON \& LABUZA (1977) encontraram, na dieta dos estudantes universitários estudados, consumo médio elevado de cálcio $(111 \%$ de adequação). Apesar disso, os autores chamam atenção para o fato de que um número considerável da sua população consumiu menos de $60 \%$, comentando que provavelmente esse fato se deva ao baixo consumo de produtos lácteos observado. Em nosso estudo, como já referimos, o consumo médio de leite e derivados foi de $197,5 \mathrm{~g}$ por dia.

O nutriente cujo consumo parece estar mais deficiente entre nossas estudantes é o ferro. De fato, podemos observar, na figura 1, ter sido esse nutriente o que apresentou o menor nível de adequação. O problema assume proporções mais graves ao verificarmos que $80 \%$ das estudantes consumiram menos de $90 \%$ da recomendação (tabela 4).

WHITE (1970), ao analisar a deficiência de ferro em mulheres jovens, revisando mais de 60 investigações realizadas nas décadas de 1950 e 1960, verificou que a média do consumo do mineral variou de 10 a $12 \mathrm{mg}$ por dia; dos 60 trabalhos analisados, somente 12 apresentaram dados de ingestão média de ferro superior a $12 \mathrm{mg}$ por dia. Outros estudos mais recentes confirmam o baixo conteúdo de ferro na dieta de estudantes universitários do sexo feminino (MAFKAWA et alii, 1977; ISHIGAKI \& SUZUKI, 1975; DRISKELL et alii, 1979; TALEBANY, 1981).

OSTRON \& LABUZA (1977) registraram média bem inferior (56\% de adequação) à encontrada no presente estudo, sugerindo que a deficiência de ferro é um problema em potencial, mesmo na população universitária feminina.

Uma avaliação mais criteriosa deveria considerar o tipo de dieta, a origem do ferro e a presença de fatores que favorecem ou que inibem a absorção do nutriente. Como sabemos, o ferro dietético proveniente das carnes é mais bem absorvido que o proveniente das fontes de origem vegetal; este último é absorvido melhor quando ingerido simultaneamente ao de origem animal. Sabemos, também, que a ingestão concomitante de ácido ascórbico aumenta a biodisponibilidade do ferro dietético favorecendo sua absorção (LAYRISSE et alii, 1974; LAYRISSE et alii, 1968; LAYRISSE et alii, 1969; RATHEE \& PRADHAN, 1980; INTERNATIONAL NUTRITIONAL ANEIMIA CONSULTATIVE GROUP, 1977; HALLBERG, 1974).

Em nosso estudo, constatamos que $40 \%$ do ferro consumido provinha de alimentos animais, principalmente carnes, o que, quando comparado com os estudos do INTERDEPARTAMENTAL COMMITTEE ON NUTRITION FOR NATIONAL DEFENSE (1969) e da FUNDAÇÃO INSTITUTO BRASILEIRO DE GEOGRAFIA E ESTATISTICA (1982), poderia representar proporção bastante razoável, dado que esses estudos revelaram ser a ingestão de ferro de diversas regiões estudadas, em grande parte, de origem vegetal, variando em média de 70 a $90 \%$. 


\section{CONCLUSÕES}

Sabemos que em qualquer estudo dietético, são inerentes as super e/ou subestimativas do consumo de nutrientes, uma vez que os cálculos se baseiam em tabelas de composição alimentar, geralmente resultado de compilações, podendo, portanto, não refletir exatamente o conteúdo nutritivo dos alimentos produzidos e consumidos naquela região; não levam em consideração as perdas nutricionais que podem ocorrer durante a manipulação e preparo dos alimentos nem os possíveis erros cometidos por ocasião da pesagem e/ou conversão do peso dos alimentos cozidos em crus.

Apesar dessas limitações, eles constituem uma valiosa fonte de informação do consumo alimentar atual do grupo em estudo.

Os achados do presente estudo mostram claramente o perigo de trabalharmos com dados de consumo médio; no que toca às vitaminas $\mathrm{A}$, $\mathrm{B}_{1}, \mathrm{~B}_{2}, \mathrm{C}$ e niacina, apesar do consumo médio adequado, quase adequado ou mesmo excessivo, grande parte da população estudada apresentava consumo deficiente, mascarado pelo consumo excessivo de uma parte da população, particularmente no que toca à vitamina $A, B_{1}$ e $B_{2}$.

Quanto ao ferro e ao cálcio, podemos considerar que a ingestão deficiente é quase generalizada, constituindo, com a vitamina $A$ e $B_{1}$ as quatro principais deficiências nutricionais observadas na alimentação da população estudada no presente trabalho, quando comparada com as recomendações nutricionais.

FUJIMORI, E.; SHIMA, H.; SALUM, M.J.L. Food intake of female university students. Rev. Esc. Enf. USP, São Paulo, 20(2):115-124, 1986.

Weighted food intake was the precedure adapted by the authors in order to find aut the nutritional pattern of female university students. The results revealed vitaminic-mineral deficiency (mainly of calcium, iron and thiamine) in relation to the recommended dietary allowances.

\section{REFERENCIAS BIBLIOGRAFICAS}

1. BURTON, B.T. Tabelas de composiçăo dos alimentos. In: - Nutrição humana. São Paulo, McGraw-Hill, 1979. p.544-67.

2. DRISKELL, J.A.; KEITH, R.E.; TANGNEY, C.C. Nutritional status of white college students in Virginia. J. Amer. dlet. Ass., Chicago, 74(1) :32-5, 1979.

3. FOOD AND NUTRITION BOARD. Recommended Dietary Allowances. 8th rev. Washington, National Academy of Sciences - National Research Council, 1974.

4. Recommended Dietary Allowances. 9th rev. Washington, National Academy of Sciences - National Research Councll, 1980.

5. FUNDACAO INSTITUTO BRASILEIRO DE GEOGRAFIA E ESTATISTICA. Estudo Naclonal da despesa familiar - ENDEF: tabelas do composição dos alimentos. Rio de Janeiro, IBGE, 1977. 201p.

6.

Perfil estatistico de crianças o mäes no Brasil : aspectos nutricionais, 1974-1975. Rio de Janeiro, IBGE, UNICEF, 1982. 267p. 
7. GRANT, J.P.; CUSTER, P.B.; THURLOW, J. Técnicas atuais para avaliação nutricional. Clin. cir. Amer. Norte, Rio de Janeiro, 61(3):441-69, 1981.

8. HALLBERG, L. The pool concept in food iron absorption and some of its implications. Proc. Nutr. Soc., London, 33(3):285-91, 1974.

9. INSTITUTO DE NUTRICION DE CENTRO AMERICA Y PANAMA. Recomendaciones nutricionalis diarias para las poblaciones de Centro America y Panamá. Washington, 1966. p.75-6 (Publicación científica, 136).

10. INTERDEPARTAMENTAL COMMITTEE ON NUTRITION FOR NATIONAL DEFENSE. Northest Brazil: nutrition survey - a report. Apud GANDRA, Y.R. Iron deficiency anemia in Latin American and Caribbean populations. In: PAN AMERICAN HEALTH ORGANIZATION. Iron metabolism and anemia. Washington, 1969. p.56-64. (Scientific Publication, 184).

11. INTERNATIONAL NUTRITIONAL ANEMIA CONSULTATIVE GROUP. Guidelines for the eradication of iron deficiency anemia: a report. New York, Nutrition Foundation, 1977. 29p.

12. ISHIGAKI, S. \& SUZUKI, J. A study on nutrient intake conditions and caloric consumption of girl students. Jap. J. Nutr., Tókio, 33(2):79-83, 1975.

13. KAGAKU GIDYUTSU CHO SHIGEN CHOSA KAI. Nippon shokuhin seibunhyó. Tókio, 1979. 217p.

14. LAYRISSE, M.; COOK, J.D.; MARTINEZ-TORRES, C.; ROCHE, M.; KUHN, I.N.; WALKER, R.B.; FINCH, C.A. Food iron absorption: a comparison of vegetable and animal foods. Blood, New York, 33(3):430-43, 1969.

15. LAYRISSE, M.; MARTINEZ-TORRES, C.; ROCHE, M. Effect of interation of various foods on iron absorption. Amer. J. clin. Nutr., New York, 21(10):1175-83, 1968.

16. LAYRISSE, M. : MARTINEZ-TORRES, C.: GONZALEZ, M. Measurement of the total dally dietary iron absorption by the extrinsic tag model. Amer. J. clin. Nutr., New York, 27(2):152-62, 1974.

17. MAEKAWA, M.; YAGURAMAKI, K.; TERUKO, M.; FUKINO, Y.; ITO, R.; MARIOKA, K. A nutritional survey and the daily schedule of college girls. Jap. J. Nutr., Tókio, 35(6):307-14, 1977.

18. MARCONDES, E. Normas para o diagnóstico e a classificação dos distúrbios do crescimento e da nutrição - última versão. Pediatria, São Paulo, 4(4):307-26, 1982.

19. NAKamaf, D.D. Perfil do estudante de enfermagem. São Paulo, 1976 . (Dissertaçăo de Mestrado - Escola de Enfermagem da USP).

20. ORGANIZACION MUNDIAL DE LA SALUD. Prevención de la mortalidad y la morbidad perinatales. Genebra, 1970. 69p. (Séries de Informes Técnicos, 457).

21. OSTRON, S. \& LABUZA, T.P. Analysis of a seven-day diet survey of college students. Food Technology, Chicago, 31(5):68-76, 1977.

22. RATHEE, S. \& PRADHAN, K. Effect of ascorbic acid on availability of iron from an egg based whole day diet of college girls. Indian J. Nutr. Diet., Coimbatore, 17(3): 90-4, 1980.

23. TALEBANY, L.J. Dietary evaluation of college students. J. Fac. Med., Baghdad, Baghdad, 23(2):207-16, 1981.

24. WHITE, H.S. Iron deficiency in young women. Amer. J. publ. Hth., New York, 60(4): 659-65, 1970. 